\title{
Reduction of the hydrophobic attraction between charged solutes in water
}

\author{
J. Dzubiella* and J.-P. Hansen \\ University Chemical Laboratory, Lensfield Road, Cambridge CB2 1EW, United Kingdom
}

(Dated: November 6, 2018)

\begin{abstract}
We examine the effective force between two nanometer scale solutes in water by Molecular Dynamics simulations. Macroscopic considerations predict a strong reduction of the hydrophobic attraction between solutes when the latter are charged. This is confirmed by the simulations which point to a surprising constancy of the effective force between oppositely charged solutes at contact, while like charged solutes lead to significantly different behavior between positive and negative pairs. The latter exhibit the phenomenon of "like-charge attraction" previously observed in some colloidal dispersions.
\end{abstract}

PACS numbers: 82.70.Uv,87.16.Ac,61.20Ja,68.08.Bc

When apolar solutes are dispersed in water, they exert effective, solvent induced forces on each other, generally referred to as hydrophobic attraction. For large solutes, say globular proteins, the mechanism for hydrophobic interactions can be traced back to solvent depletion (or "drying") from the volume bounded by opposite surfaces of the solutes [1, 2]. This mechanism, which is reminiscent of polymer-induced depletion attraction between colloidal particles on larger (mesoscopic) scales 3], is most pronounced when the solvent is near liquid-vapour coexistence 2]. However, many biomolecular solutes are charged, and in this work we present evidence, based on extensive Molecular Dynamics (MD) simulations, that the electric field due to the charges carried by the solutes leads to a considerable reduction of the hydrophobic attraction.

Such a reduction may be qualitatively understood from macroscopic considerations. It is a well-known fact that a polar liquid, like water, will rise inside a vertical condenser to minimize the overall electrostatic energy (the rise being limited by gravity on a macroscopic scale) [4]. Transposing this situation to the nanometric scale, consider two parallel plate-like solutes of area $A$, separated by a distance $D$, inside a polar solvent of relative dielectric permittivity $\epsilon$, carrying opposite surface charges $\pm \sigma$. Neglecting edge effects, the electric field between the plates is $E_{0} / \epsilon$ with $E_{0}=\sigma / 2 \epsilon_{0}$. We require the difference in the grand potential between the situations where the liquid solvent $(l)$ or its vapour $(g)$ fill the volume $A D$ between the two plates:

$$
\Omega_{\alpha}=-P_{\alpha} A D+2 \gamma_{w \alpha} A+\frac{1}{2} \epsilon_{0} \frac{E_{0}^{2}}{\epsilon_{\alpha}} A D ; \quad \alpha=l, g
$$

where $P_{\alpha}$ is the pressure of phase $\alpha$ and $\gamma_{w \alpha}$ the surface tension between phase $\alpha$ and the plate ("wall"). Consider a state close to phase coexistence at temperature $T$, and let $\delta \mu=\mu-\mu_{\text {sat }}$ be the positive deviation of the chemical potential from its saturation value. Expanding the $P_{\alpha}$ to linear order in $\delta \mu$ around their common value at

*e-mail address: jd319@cam.ac.uk saturation, one easily arrives at the following expression for the difference in grand potentials per unit area:

$$
\begin{aligned}
\frac{\Omega_{l}-\Omega_{g}}{A} & =\left(\rho_{g}-\rho_{l}\right) \delta \mu D+2\left(\gamma_{w l}-\gamma_{w g}\right) \\
& +\frac{\epsilon_{0}}{2} E_{0}^{2}\left(\frac{1}{\epsilon_{l}}-\frac{1}{\epsilon_{g}}\right) D
\end{aligned}
$$

At the "drying" transition between the plates, $\Omega_{l}-\Omega_{g}=$ 0 and $\gamma_{w l}-\gamma_{w g}=\gamma_{l g} \equiv \gamma$. Since $\rho_{g} \ll \rho_{l}$, and $\epsilon_{l} \equiv \epsilon \gg$ $\epsilon_{g} \simeq 1$, eq. (2) yields the following expression for the critical distance $D_{\text {c }}$ between the plates at which drying occurs:

$$
D_{\mathrm{c}} \simeq \frac{2 \gamma}{\rho_{l} \delta \mu+\frac{\epsilon_{0}}{2} E_{0}^{2}}
$$

For strong electric fields, $\left(E_{0} \lesssim 10^{10} \mathrm{~V} / \mathrm{m}\right.$ corresponding to surface charges $\left.\sigma \lesssim e / \mathrm{nm}^{2}\right)$, the electrostatic term in the denominator is typically 10 times larger than the $\delta \mu$ term in the vicinity of gas-liquid coexistence, and leads to values of $D_{\text {c }}$ of the order of a few $\AA$. This strong reduction of $D_{\mathrm{c}}$ hints at a considerable weakening of the hydrophobic interaction between two solutes when the latter are charged. Note that within our macroscopic model this reduction is due to the overall electric field, not to any Hydrogen-bonding of the solvent molecules to hydrophilic "patches" on the solute surface.

In order to confirm the qualitative prediction of the schematic model, we have carried out extensive MD simulations of two spherical solutes immersed in a bath of SPC/E water molecules [5]. The solutes are spheres of radius $R$ which repel the solvent molecules by a repulsive $\epsilon(r-R)^{-12}$, where $r$ is the distance from the solute center to the oxygen atom of a water molecule; the energy scale $\epsilon$ is chosen such that the $\mathrm{O}$ atom experiences an energy $k_{\mathrm{B}} T$ at a distance of $r-R=1 \AA$ from the solute surface. The simulation cell is a cube of length up to $L=40 \AA$, containing up to 2000 water molecules, depending on the solute size; solutes are placed at fixed positions on the body diagonal of the simulation cell. The box dimensions are chosen such that the surface to surface distance to the nearest image solute is at least $20 \AA$.

The MD simulations are carried out with the DLPOLY2 package [6], using the Verlet algorithm [7], 

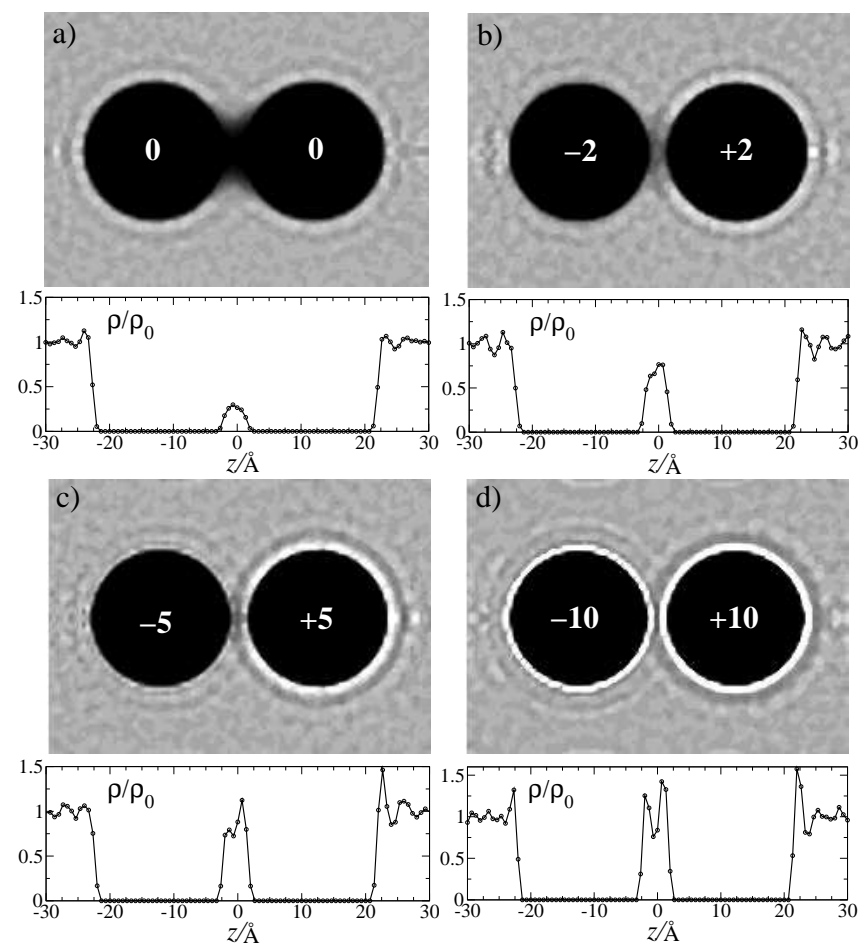

FIG. 1: Density profiles of the water molecules around two neutral spherical solutes of radius $R=10 \AA$ (a) and two oppositely charged spherical solutes of radius $R=10 \AA$ carrying a charge $\pm q e$ with (b) $q=2$, (c) $q=5$, and (d) $q=10$. The surface-to-surface distance in all cases is $s=4 \AA$. In the contour plots dark regions show low density regions while high densities are plotted bright. The panels below the contour plots show the water density $\rho$ scaled with water bulk density $\rho_{0}$ in a cylinder of radius $R_{c}=5 \AA$, coaxial with the center-to-center line of the solutes.

with a timestep of $2 \mathrm{fs}$. The Berendsen barostat and thermostat [8] were used to maintain the SPC/E water at a pressure of 1 bar and a temperature $T=300 \mathrm{~K}$. All electrostatic interactions were calculated using particle-mesh Ewald summations [9]. We first consider the case of uncharged solutes. The water density profile is illustrated in frame (a) of Fig. 1 for the case of solutes of radius $R=10 \AA$ and a surface-to-surface distance along the $z$-axis joining the centers $s=4 \AA$. The upper part of the frame shows a density contour plot coded by variable shades of grey. The lower part shows density profiles along the center-to-center to axis $z$, averaged over a coaxial-cylindrical volume of radius $5 \AA$. The density profiles show a considerable depletion of the solvent within a radial distance of $5 \AA$ from the center-to-center axis, reminiscent of the observations of Wallquist and Berne for flatter solutes [1]. As the surface-to-surface distance $s$ is increased for fixed radius $R$, the water molecules penetrate into the region between opposite solute surfaces, as signalled by a rapid increase of the central peak (around

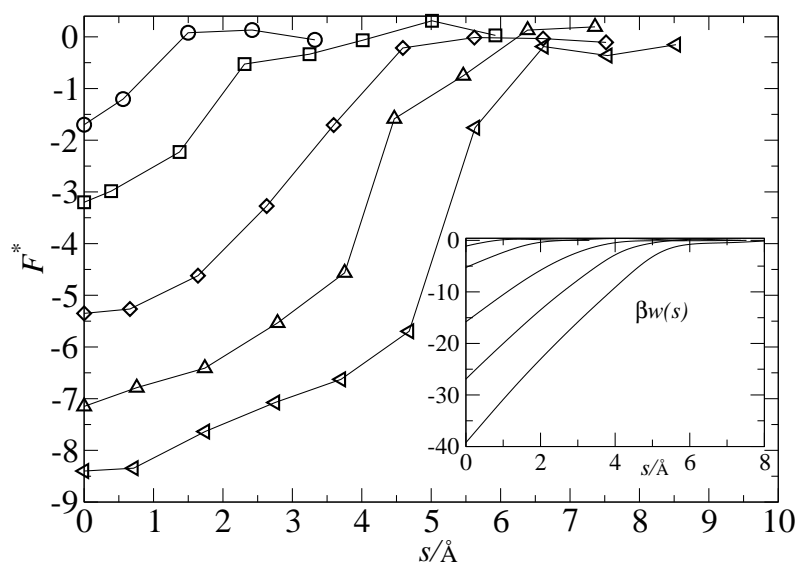

FIG. 2: Simulation results (symbols) of the mean force, $F^{*}=\beta F \AA$, between two neutral $(q=0)$ spheres in $\mathrm{SPC} / \mathrm{E}$ water. Error bars are omitted for clarity; the error is estimated to be $\Delta F^{*}=0.4$, comparable to the symbol size. The lines are guide to the eye. Results are plotted for solute radii $R=$ $3 \AA$ (circles), $R=5 \AA$ (squares), $R=8 \AA \quad$ (diamonds), $R=10 \AA$ (triangles pointing up), and $R=$ $12 \AA$ (triangles pointing left). The inset shows the integrated force (potential of mean force) in obvious order.

$z=0$ ) in the density profiles. When $s \approx 6.5 \AA$, the solvent layers around an isolated solute are hardly disturbed by the presence of the other solute.

The mean effective force acting on each of the solutes in the presence of the second at a surface-to-surface distance $s$ is calculated by averaging the total force due to all solvent molecules over the configurations generated by MD runs extending over typically $1 \mathrm{~ns}$. This average force obviously goes to zero at large distances $s$ and for symmetry reasons, it is directed along the center-to-center axis. Examples for several radii $3 \AA \leq R \leq 12 \AA$ are shown in Fig. 2. The largest radii are of the order of the size of small globular proteins or of oil-in-water micelles. As expected from a depletion mechanism, the force is attractive and its contact values and range increase with $R$. The potentials of mean force $w(s)$ may be calculated for each $R$ by integrating the force. The resulting potentials are shown in the inset to Fig. 2. They closely resemble results obtained for polymer-induced depletion potentials between spherical colloids, albeit on different length and energy scales [1, 10]. Note that the force at contact, $F(0)$, scales roughly with $R$. This may be rationalized by a simple consideration of the potential of mean force for plates $(R=\infty)$ at contact, $w(0)=-2 \gamma$ (where $\gamma$ is now the plate-solvent surface tension which differs little from the liquid-gas surface tension [1] ) and an application of the Derjaguin approximation, valid for weakly curved substrates (i.e. large $R$ ) [10]; this leads to the estimate $F(s=0)=-2 \pi R \gamma$, which indeed predicts linear scaling; the value $\gamma \simeq 0.05 \mathrm{~J} / \mathrm{m}^{2}$, extracted 


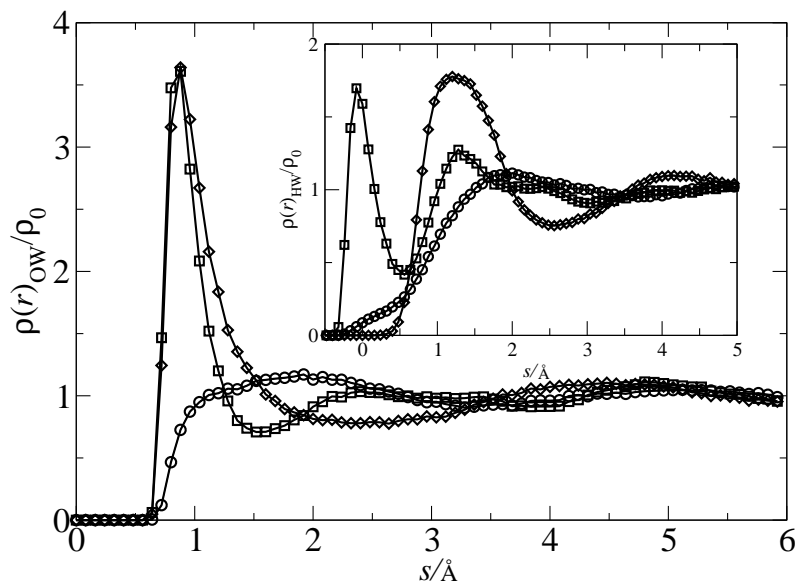

FIG. 3: Density profiles of water oxygen and hydrogen atoms (inset) around one isolated solute with radius $R=10 \AA$ and central charge $q=0$ (circles), $q=-10$ (squares), and $q=10$ (diamonds).

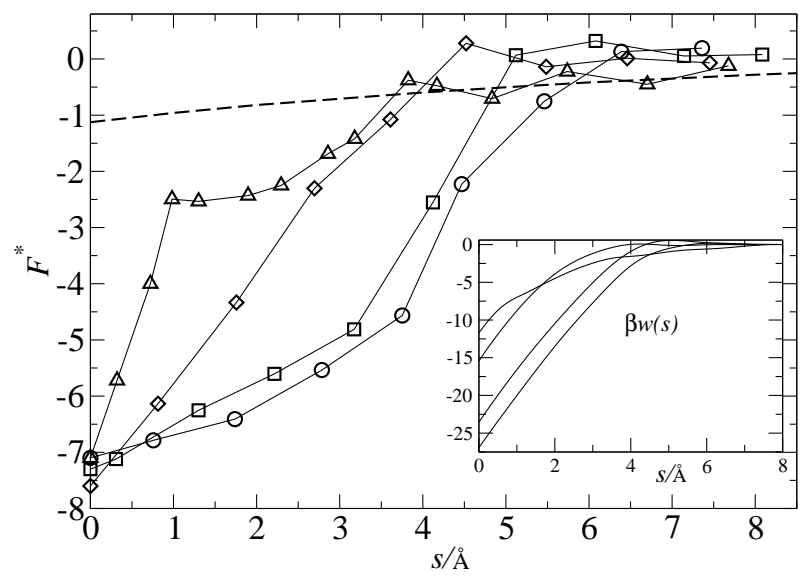

FIG. 4: Same as Fig. 2 but now the mean force, $F^{*}=$ $\beta F \AA$, is for oppositely charged spheres of $R=10 \AA$ and different central charges $\pm q e$ are shown: $q=0$ (circles), $q=2$ (squares), $q=5$ (diamonds), $q=10$ (triangles up). The dashed line represents the electrostatic force between 2 periodically repeated solutes with opposite charges $q= \pm 10$ in a continuous solvent with permittivity $\epsilon=80$. The inset shows the resulting potentials of mean force; the contact values $w(s=0)$ increase with $q$.

from the MD value of $F(0)$ for the largest $R$, is reasonably close to the liquid-vapour surface tension of water under normal conditions $\left(\gamma=0.073 \mathrm{~J} / \mathrm{m}^{2}\right)$. The agreement must be considered quite satisfactory in view of the roughness of the estimate.

We now turn to the charged solutes. Frames (b)-(d) in Fig. 1 show water density profiles in the vicinity of two spheres carrying opposite electric charges $\pm q e$ at their center (opposite charges ensure overall charge neutrality without any need for counterions). As $q$ increases from zero (frame (a)), water is seen to penetrate between the two solutes, the central peak around $z=0$ in the density profiles increases rapidly and its amplitude reaches roughly the bulk density of water when $q=10$. Note that this central peak is asymmetrically split, indicating the presence of two hydration layers which differ somewhat depending on their association with the anionic or cationic solute. This difference is also evident in the contact values of the outside surfaces of the solutes, and is a consequence of the different arrangements of the water dipoles around the solutes induced by the local electric fields. The asymmetry of the profiles can be rationalized by inspecting the density profiles of $\mathrm{O}$ and $\mathrm{H}$ atoms around isolated solutes, plotted in Fig. 3. The hydration shell is more sharply defined around the cationic than around the anionic solute. The water dipoles tend to point radially away from the cation, while the opposite configuration is more favourable around anions. Note also that driving water into a narrowly confined region under the action of a strong electric field (here between oppositely charged solutes) is an effect reminiscent of that observed in recent MD simulations of ion permeation of hydrophobic nanopores [12].

The resulting mean forces between solutes are plotted for $q=0,2,5$ and 10 , as functions of the surface-tosurface distance $s$ in Fig. 4 together with corresponding potentials of mean force. The mean force includes the direct Coulomb interaction between the two solutes (with proper account for the periodic images), which is in fact an order of magnitude larger that the total mean force. At large distances hydrophobic interactions become negligible and the force should tend to $-q^{2} e^{2} /\left(4 \pi \epsilon_{0} \epsilon r^{2}\right)$, where $r=2 R+s$ and $\epsilon$ is the dielectric permittivity of bulk SPC/E water; the corresponding curve is also shown in Fig. 4.

The most striking result illustrated in Fig. 4 is the near independence of the force at contact, $s=0$, with respect to solute charge. From the density profiles in Fig. 1 the hydrophobic attraction is expected to be reduced but this reduction is almost exactly compensated by the Coulomb attraction between solutes in the presence of the solvent. As $q$ increases, the initial slope of the effective force increases. The potential of mean force (shown in the inset of Fig. 4) exhibits a contact value which increases with $q$, indicating that the reduction of hydrophobic attractive free energy and the reduction of dielectric screening due to restructuring of water by the strong electric field of the solutes clearly outweigh the increase in bare Coulomb attraction between the latter. Simulations calculating the forces at and near contact for $q=7$ and $q=15$, not shown in Fig. 4, confirm this trend. Note that the potential of mean force curve for $q=10$ shows more long range attraction as compared to the smaller $q$ data due to the increased electrostatic attractive interaction. The eye-catching kink in the force for $q=10$, at a distance $s \approx 1 \AA$ is reproducible, and is probably related to the pronounced shell structure of water molecules around highly charged solutes, illustrated 


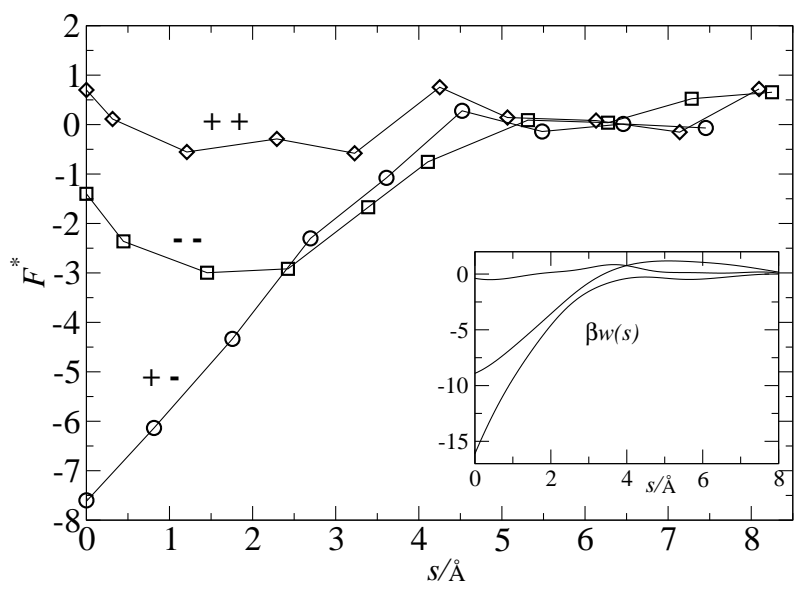

FIG. 5: Same as Fig. 2 but now the mean force, $F^{*}=\beta F \AA$, is for different signs of the charged spheres of radius $R=10 \AA$ and fixed central charges $q e= \pm 5 e$ are shown: oppositely charged (+ -,circles), equally charged with $q=-5(--$ ,squares), equally charged with $q=5$ ( ++ ,diamonds).

in Fig. 3. While for neutral (and weakly charged) solutes, the $\mathrm{O}$ and $\mathrm{H}$ density profiles show little structure, they are sharply peaked at a distance $s \approx 1 \AA$ of the $\mathrm{O}$ atoms from the solute surface. This would lead to a complete shared hydration layer, and consequently to a kink in the force versus distance curve, between 2 flat solutes separated by $s=2 \AA$. This critical separation is shifted to shorter distances due to the curvature of spherical solutes.

In view of this delicate balance between various interactions, we have also examined the case of equally charged solutes. In this case monovalent counterions $\left(\mathrm{Na}^{+}\right.$or $\left.\mathrm{Cl}^{-}\right)$were included to ensure overall charge neutrality. The situation is summarized in Fig. 5 for solutes of radius $R=10 \AA$ and charge $q= \pm 5$. The water density profiles (not shown here) are symmetric with respect to $z=0$ for equally charged solutes, but differ substantially when going from a pair of anions to a pair of cations. In the latter case water is much more structured into well defined hydration shells, as shown in Fig. 3. This difference is reflected in the effective forces and potentials shown in Fig. 5. While the force and potential of mean force are practically zero at all distances in the case of cationic solutes, anionic solutes attract each other significantly, showing that residual hydrophobic attraction overcomes the repulsion between like charges, which may be more efficiently reduced by the local permittivity of water in the immediate vicinity of the anionic solutes.

To summarize, we have shown that electric charges carried by nanometer-scale solutes have a profound influence on hydrophobic interactions. Our MD simulations confirm that for neutral solutes hydrophobic attraction is intimately linked to solvent depletion, and the contact value of the hydrophobic force is directly related to the surface tension of the pure solvent. Solvent depletion is suppressed by the electric field due to any charge carried by the solute, but for oppositely charged solutes the resulting loss of hydrophobic attraction is compensated by their mutual Coulomb attraction, leading to a nearly constant contact force. The effective force between equally charged solutes depends on the sign of their charge. Anionic solutes exhibit a striking "like-charge attraction", a subject of considerable debate in the field of mesoscopic charge-stabilized colloids [13]. Coulombic and hydrophobic effects are intimately entangled, and their inter-relation is expected to play an important role in the analysis of protein aggregation and related biomolecular mechanisms.

The authors are grateful to R. Evans and H. Löwen for useful clarifications. JD acknowledges the financial support of EPSRC within the Portfolio Grant RG37352.
[1] A. Wallquist and B. J. Berne, J. Phys. Chem. 99, 2893 (1995).

[2] K. Lum, D. Chandler, and J. D. Weeks, J. Phys. Chem. B 103, 4570 (1999).

[3] C. N. Likos, Phys. Rep. 348, 267 (2001).

[4] L. D. Landau, E. M. Lifshitz, and L. P. Pitaevski, Electrodynamics of Continious Media (Butterwort-Heinemann, 1993), second edition.

[5] H. J. C. Berendsen, J. R. Grigera, and T. P. Straatsma, J. Phys. Chem. 91, 6269 (1987).

[6] W. Smith and T. R. Forester (1999), the DLPOLY_2 User Manual.

[7] D. Frenkel and B. Smit, Understanding Molecular Simulation: From Algorithms to Applications (Academic Press, 1996).
[8] H. J. C. Berendsen, J. P. M. Postma, W. F. van Gunsteren, A. DiNola, and J. R. Haak, J. Chem. Phys. 81, 3684 (1984).

[9] U. Essmann, L. Perera, M. L. Berkowitz, T. Darden, and H. L. L. G. Pedersen, J. Chem. Phys. 103, 8577 (1995).

[10] A. A. Louis, P. G. Bolhuis, and J. P. Hansen, J. Chem. Phys 117, 1893 (2002).

[11] D. M. Huang, P. L. Geissler, and D. Chandler, J. Phys. Chem. B 105, 6704 (2001).

[12] J. Dzubiella, R. J. Allen, and J. P. Hansen (2003), to be published.

[13] J. P. Hansen and H. Löwen, Ann. Rev. Phys. Chem. 51, 209 (2000). 